\section{Dangerous surgical scavenger hunt: the complicated course of a patient with left ventricular assist device and end-stage renal disease undergoing reconstructive flap surgery}

\author{
M Freundt, ${ }^{1,2}$ A Haneya, ${ }^{1,3}$ C Schmid' and S Hirt'
}

\begin{abstract}
Patients with left ventricular assist devices (LVADs) who develop stage IV sacral pressure sores (SPS) have an increased procedural risk. We present the complications, including severe intra- and postoperative bleeding, diarrhea with metabolic acidosis, volume loss and acute on chronic renal failure, flap dehiscence and late LVAD outflow cannula thrombosis, in a 54-year-old male who underwent diverting ileostomy (DI) and subsequent fasciocutaneous flap (FCF) surgery for stage IV SPS while supported with an LVAD. Our experience suggests that, despite continuous heparinization, life-threatening thrombotic complications, such as device clotting, can occur. Therefore, the benefit of intervention has to outweigh the risk of bleeding, which should be managed with meticulous surgical technique and substitution of red blood cells rather than the reversal of heparinization or the substitution of clotting factors. Continuation of double anti-platelet therapy should also be considered.
\end{abstract}

\title{
Keywords
}

left ventricular assist device; flap surgery; intra-abdominal surgery; end-stage renal failure; complication

\section{Introduction}

Left ventricular assist devices (LVADs) are an established therapeutic strategy in patients with end-stage heart failure. LVADs require continuous triple anticoagulation (AC) to prevent thromboembolic complications and device clotting. ${ }^{1}$ Even though a significant improvement in functional status has been shown, the clinical course can be long. A known complication of long-term hospitalization is sacral pressure sores (SPS), which contribute to pain, infectious complications and increased risk of death. ${ }^{3}$ As previously shown, invasive type 3 and 4 SPS are best treated surgically. ${ }^{4}$ To prevent fecal contamination, the placement of a diverting ileostomy (DI) usually precedes reconstructive surgery.

\section{Case Description}

A 54-year-old male underwent LVAD (HeartWare ${ }^{\oplus}$, International Inc., Framingham, MA) implantation as a bridge to transplant for severe ischemic cardiomyopathy with prolonged hospitalization. Five by eight by ten centimeter stage IV SPS developed. Five months after LVAD implantation, the clinical situation was stable and the single limiting issue remained the SPS (Figure 1).

Warfarin and clopidogrel were discontinued and a heparin drip with the goal of a partial thromboplastin time (PTT) of 60-80 seconds was initiated. Aspirin (ASA) $100 \mathrm{mgs}$ daily was continued. Uncomplicated laparoscopic DI placement was performed without significant blood loss despite continuation of the heparin

\footnotetext{
'Dept. Cardiothoracic Surgery, University Hospital Regensburg, Germany

2Exempla Saint Joseph Hospital, Internal Medicine Residency Program, Denver, CO, USA

${ }^{3}$ Department of Cardiovascular Surgery, University of SchleswigHolstein, Campus Kiel, Kiel, Germany
}

\section{Corresponding author:}

Miriam Freundt, MD

Exempla Saint Joseph Hospital

Internal Medicine Residency Program

I835 Franklin Street

Denver, CO, 80218, USA

Email: miriamfreundt@me.com 


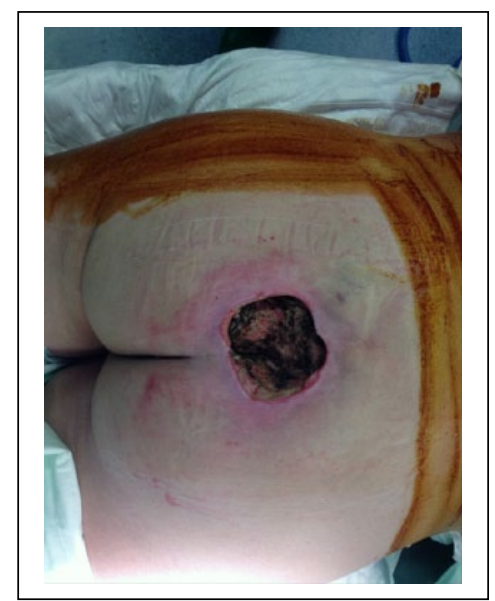

Figure I. Sacral pressure sore preoperatively.

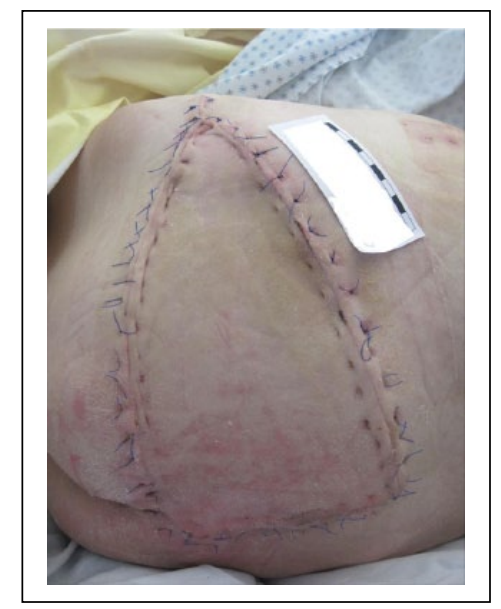

Figure 2. Fasciocutaneous flap.

drip. General anesthesia (GA) was tolerated well without hemodynamic instability. On postoperative day (POD) 3, the patient suffered small bowel obstruction (SBO), resistant to conservative measures, which prompted emergent take-back to the OR on POD 6 . Exploratory laparoscopy had to be converted to median laparotomy due to extensive diffuse bleeding and poor visibility. Small bowel loops appeared twisted around the ileostomy, which was then disconnected and reinserted. Diffuse oozing prolonged surgery, but stopped after the PTT was kept at 60-70 sec and several units of packed red blood cells (PRBC) had been transfused. Fresh frozen plasma and protamine were avoided due to the fear of the LVAD clotting. The abdomen was closed and recovery was uneventful, with proper DI function. Later, non-infectious diarrhea caused severe metabolic acidosis and volume loss. In addition, patient suffered deterioration of chronic kidney disease, becoming dependent on hemofiltration.

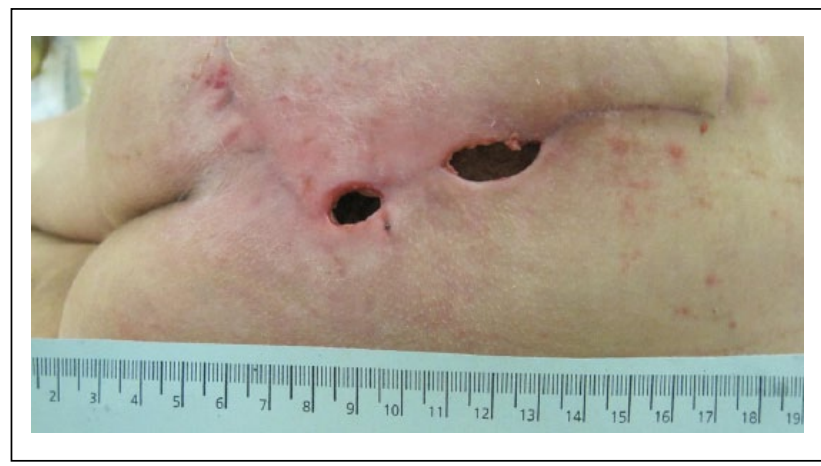

Figure 3. Wound dehiscence.

On POD 23, excision of the SPS, debridement and coccygectomy were performed in the prone position with GA and on a heparin drip and ASA. Extensive diffuse bleeding prompted the transfusion of 4 PRBC and packing of the wound with resolvable hemostyptica and surgical pads rather than vacuum sponge therapy. The bleeding eventually stopped, with strict control of the PTT at 60-70 $\mathrm{sec}$ and local pressure. At this point, ASA was discontinued. On POD 27, ipsilateral VY-FCF surgery was performed, significant for extensive diffuse bleeding, requiring the substitution of 2 units of PRBC, placement of an extra drain and several strips of resolvable hemostyptica underneath the flap. A small hematoma resolved with conservative management, the wounds healed properly (Figure 2) and ASA was restarted.

Four weeks later, the LVAD flow suddenly dropped to $1.5 \mathrm{~L} / \mathrm{min}$. A computed tomography (CT) scan revealed thrombosis of the LVAD outflow cannula. Thrombolysis with tirofiban remained unsuccessful and severe epistaxis limited further attempts. Deteriorating hemodynamics required endostent placement into the outflow cannula and the LVAD flow immediately came back to $3.5 \mathrm{~L} / \mathrm{min}$. The day after, two $2-\mathrm{cm}$ wound defects (Figure 3) of the FCF, with spontaneous discharge of fresh, non-infected hematoma occurred, which was managed conservatively. Eventually, the patient suffered a major intracerebral hemorrhage and expired.

\section{Discussion}

With an increasing number of patients receiving LVADs, the need for surgical procedures on support systems is increasing. Guidelines for perioperative management haven't yet been established. A previous study identified major postoperative bleeding as the main problem in smaller procedures, such as cholecystectomy, appendectomy and tracheostomy, where heparin was held perioperatively without clotting complications. ${ }^{6}$ Another study claimed that patients with 
LVADs tolerated smaller procedures with perioperative discontinuation of AC with tolerable thromboembolic risk. ${ }^{7}$ Contrary to these cases with favorable outcomes, Ficke et al. describe a patient with LVAD, who underwent an uneventful cystoscopy, but, shortly thereafter, went into cardiac arrest with fatal outcome. ${ }^{8}$

The challenge in our case was the need for consecutive major surgical procedures. We chose perioperative continuation of heparin and ASA to avoid thromboembolic events in acceptance of increased intra- and postoperative bleeding. The strict PTT range of $60-80 \mathrm{sec}$ was insufficient and clotting occurred as a late complication. During a period of severe hemorrhagic complications, ASA had to be withheld and was then not continued, which might have contributed. We decided on pro fecal diversion to avoid contamination and promote wound healing, even if this added a procedure, risking further complications. For reconstruction, an ipsilateral VY-FCF was chosen over a musculocutaneous flap to limit bleeding. The soft tissue defect occurred unusually late and seems likely related to local pressure during lying supine on the unpadded angiography table. Fatal intracranial bleeding occurred weeks after the intervention and seems independent.

\section{Concluding}

With the size of the SPS faced, conservative management would unlikely have resulted in satisfactory SPS healing. Surgical intervention was needed, but caused myriad subsequent complications. From our experience, it is imperative to continue heparinization and ASA during major surgery in patients with LVADs as life-threatening LVAD clotting can occur. Therefore, in these fragile patients, the benefit of intervention has to outweigh the risk of bleeding, which should be managed with meticulous surgical technique and substitution of
PRBC rather than the reversal of heparinization or the substitution of clotting factors. Continuation or, at least, the early resumption of double anti-platelet therapy should also be considered

\section{Declaration of Conflicting Interest}

The authors declare that there is no conflict of interest.

\section{Funding}

This research received no specific grant from any funding agency in the public, commercial or not-for-profit sectors.

\section{References}

1. Stulak JM, Lee D, Haft JW, et al. Gastrointestinal bleeding and subsequent risk of thromboembolic events during support with a left ventricular assist device. J Heart Lung Transplant 2014; 33: 60-64.

2. Miller LW, Pagani FD, Russell SD, et al. Use of a continuous-flow device in patients awaiting heart transplantation. NEJM 2007; 357: 885-896.

3. Allman RM. Pressure ulcer prevalence, incidence, risk factors, and impact. Clin Geriatr Med [Review] 1997; 13: 421-436.

4. Cohen N. The surgical treatment of pressure sores. Harefuah 1971; 80: 86-89.

5. Slininger KA, Haddadin AS, Mangi AA. Perioperative management of patients with left ventricular assist devices undergoing noncardiac surgery. $J$ Cardiothorac Vasc Anesth 2013; 27: 752-759.

6. Stehlik J, Nelson DM, Kfoury AG, et al. Outcome of noncardiac surgery in patients with ventricular assist devices. Am J Cardiol 2009; 103: 709-712.

7. Schmid C, Wilhelm M, Dietl K-H, Schmidt C, Hammel D, Scheld HH. Noncardiac surgery in patients with left ventricular assist devices. Surgery 2001; 129: 440-444.

8. Ficke DJ, Lee J, Chaney MA, Bas H, Vidal-Melo MF, Stone ME. Case 6-2010: Noncardiac surgery in patients with a left ventricular assist device. J Cardiothorac Vasc Anesth 2010; 24: 1002-1009. 